\title{
Transference Impedance Estimation of IEC60318 Couplers by Image Processing and Finite Element Modelling
}

\author{
Bravo, A; Ruiz, M; López, J. M; De Arcas, G; Sumpsi, M \\ Grupo de Instrumentación y Acústica Aplicada from Universidad Politécnica de Madrid \\ Ctra.Valencia Km-7, Madrid, 28031, Spain. Email:abravo@i2a2.upm.es
}

\begin{abstract}
In order to know the acoustic transference impedance of acoustic coupler described in IEC60318 standard it is necessary to make measures based on the reciprocity technique. This technique is used for microphones calibration as is defined in IEC61094-2 standard. This calibration method is complex enough to execute, and therefore it would be very interesting to look for alternative procedures that allow knowing the coupler acoustic impedance.

In this paper an acoustic impedance calibration method for type I coupler is proposed based on non invasive $X$-ray inspection, virtual instrumentation image processing application and computer simulation. This new approach requires knowing the coupler's geometry and use of finite element model approach for acoustic coupler behavior determination. In order to establish a precise mechanical model of the couplers described in IEC60318 standard it is necessary to know the geometry and key dimensions of the coupler. These dimensions are obtained with non-invasive measurement techniques, based on X-ray inspection. Then the use of a finite element model allows prediction of the coupler acoustic impedance values. This modeling is quite different from the "lumped parameter model" proposed in the IEC60318 standard. Lumped parameter modeling has limitations in its application because the dimensions of the coupler's elements are comparable with the acoustic signal wavelength. Moreover, when the sound propagates in narrow cavities and ducts the losses produced by viscosity and thermal effects must be taken into account. All these effects are not reflected with accuracy in the classic lumped model. The result of finite element simulation can provide more detailed information about the interior acoustic behavior of the coupler and therefore provide a more realistic and accurate value of acoustic impedance in order to calibrate the device. The comparison of the data obtained with this new approach and the values defined in the current version of IEC60318-1 standard (29/633/CDV) confirm the validity of the method.
\end{abstract}

Keywords - calibration, image processing, non invasive measurement system, finite element modeling, virtual measurement system, acoustic impedance measurement.

\section{INTRODUCTION}

IEC60318-1 [1] acoustic couplers are used in the calibration of the headphone included in audiometric equipment (in the electroacoustic terminology an artificial ear is the combination of one coupler and a microphone). These couplers have produced problems in the execution of audiometer calibration intercomparison activities [2]. These problems must be solved in order to make correct audiometer calibration process and thus being able to define an accurate uncertainty estimation model of aerial way [3] and [4].
For solving these disadvantages the IEC/TC29 committee has considered to define a new standard version of artificial ear that replaces the existing one [5]. In order to be able to develop this standard in a suitable way it is necessary to work on the following aspects:

- It is needed to correctly define the values of acoustic impedance that must present the artificial ear in the measurement range of interest.

- A realistic and suitable geometry has to be proposed in order to obtain these target impedance values.

- It would be interesting to identify and correct the errors due to traditional modelling based on electroacustics analogies (RLC models) in the frequency range of $20 \mathrm{~Hz}$ up to $10 \mathrm{kHz}$. The lumped model proposed in the standard is followed in the implementation for that type of couplers so the final impedance disagree the target values.

- Accomplishment of a more realistic specification in the tolerances of the objective acoustic impedance to obtain in the frequency response of the artificial ear.

In this paper some of these previous aspects will be developed starting from the following hypotheses:

1. It is necessary to know the complete external and internal geometry of a type I coupler. In this sense non-destructive inspection can be used, as it is the inspection by X-ray that will allow extracting the key dimensions of the main cavities of the coupler.

2. The correct definition and simulation by finite elements of the coupler is basic and essential in the standardization process. If the behaviour and tolerances of the impedance based on geometry are not known, it is very difficult the establishment of the basic mechanical model for the artificial ear. In [6] it was shown the attempt to construct a $2 \mathrm{D}$ model, but the results of this work were centred exclusively in showing the problems to simulate narrow tubes with losses. In this work a complete 3D model has been defined and proposed for all coupler's elements.

In detail, the paper is organized as follows. Section II provides an overview of the IEC6018 standard. In Section III, the most meaningful features of the new approach are presented. Section IV describes results from experiments are provided. 


\section{NOTES ON IEC60318 STANDARD}

The International Electrotechnical Commission (IEC) is a not-for-profit, non-governmental international standards organization that prepares and publishes International Standards for all electrical, electronic and related technologies - collectively known as "electrotechnology". IEC standards cover a vast range of technologies from power generation, transmission and distribution to home appliances and office equipment, semiconductors, fibre optics, batteries, solar energy, nanotechnology and marine energy as well as many others. The IEC also manages conformity assessment schemes that certify whether equipment, systems or components conform to its International Standards. The IEC publishes standards with the IEEE and develops standards jointly with the ISO as well as the ITU. IEC 60318 is focused on a specific kind of acoustic couplers known as acoustic couplers type I used in audiometry. This IEC standard provides with internal coupler structure and measurement methodology information.

\section{A. Structure of IEC-60318 Coupler}

The acoustic coupler that is defined in IEC-60318 standard is a mechanical stainless steel piece with external geometry of cylindrical shape. This coupler is internally composed of three interconnected acoustic cavities (two are hidden). In figure 1 , the coupler cross section representation according to IEC-60318 is shown and it is possible to see the internal cavities and the interconnection elements. In IEC-60318 standard the geometric shape and key dimensions -for cavities and interconnection elements- are not provide, which complicates the design of the coupler. Moreover, it is stated that lumped parameter approximation is not good for high frequency. In addition all commercial couplers presents screws for adjusting the acoustic impedance of the devices in the calibration process to match with expected target values.

\section{B. Measurement Method}

The impedance measurement method is based on knowing the sensitivity as frequency function for two microphones that have been calibrated in agreement with IEC61094-2 standard [7]. IEC60318 specifies that it is necessary to determine the transference impedance of the coupler in the range between $20 \mathrm{~Hz}$ and $10 \mathrm{kHz}$ using microphone sensitivities. This method presents several execution problems. There is a limited range of measurement below $100 \mathrm{~Hz}$ where the losses in the measurement process do impossible to determine with the necessary accuracy the target impedance. The measurement uncertainty is large compared with the target measurement tolerances. It has been possible to identify differences in the measurement of the impedance up to $0.5 \mathrm{~dB}$ for the same coupler in different laboratories, when the maxima tolerance is $1 \mathrm{~dB}$. In addition the values obtained in high frequency (over $5 \mathrm{kHz}$ ) show second order effects noncharacterized in the lumped model.

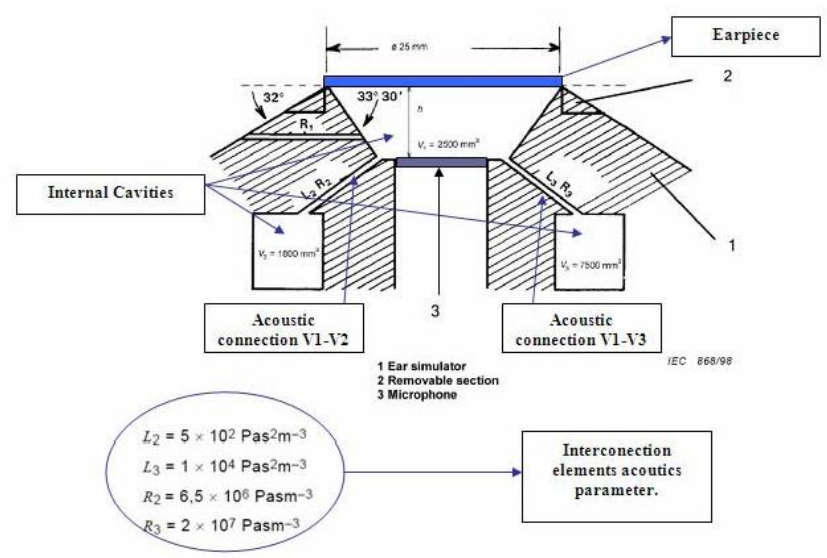

Figure 1.- IEC60318-1 type I coupler geometric and mechanic specification.

\section{PROPOSED APPROACH.}

In order to be able to make reliable and trusty finite element modelling and a later determination of the values of acoustic impedance, it becomes necessary to know the geometry and the exact dimensions of these couplers, since the IEC60318 standard leaves it as a designer election. So we propose the using of non-invasive measurement system based on X-ray inspection and image post-processing for the mechanical characterization of these standard couplers.

\section{A. Non-invasive measurement by $X$-ray inspection.}

The X-ray inspection of a commercial coupler that IEC60318 standard is made following the next steps:

- The most representative images of the coupler are extracted doing cross sections of the sample. This allows the location of the coupler's internal elements and show the geometry used in the implementation of these elements.

- A procedure for determination of dimensions based in a customized virtual instrumentation application is developed by means of image processing techniques. NI vision for Labview software module [8] is used for the image processing allowing the extraction of internal an external key coupler's dimensions. This procedure will provide us with the necessary data to:

- Reconstruct the internal cavities of the coupler.

- Generate an acoustic finite element model of the coupler (mechanical drawing and mathematical meshing).

- Compare the simulation results with the acoustic transference impedance measures obtained in a traceable laboratory under controlled environmental conditions.

For example figure 2 shows some representative images of internal element locations of an IEC60318 coupler. 


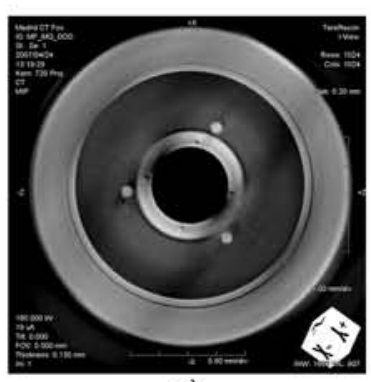

a)

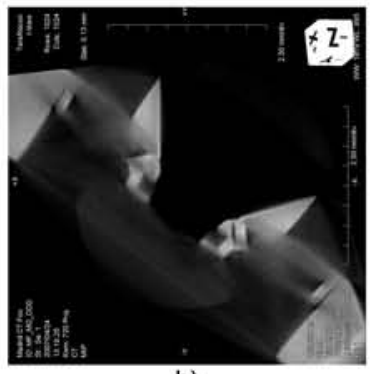

b)
Figure 2.- Non invasive X-Ray measurement system. Internal elements location of a commercial coupler a) Narrow tubes and adjustment screws b) Cavities $V_{1}, V_{2}$ and $V_{3}$.

The procedure for obtaining the key dimensions has followed these steps:

- Capture of the image to process. Several cross sections of the coupler were captured in an image video. The images determining the location of the internal cavities and narrow tubes are captured as a set of slices in XY, $\mathrm{YZ}$ and $\mathrm{XZ}$ planes. Only the most relevant images have been extracted to be processed.

- Determination of reference dimensions. Once we have selected the images to be processed it is necessary to calibrate them. These images can be affected by linear, non linear or perspective distortions that must be corrected in order to obtain the real coupler's dimensions.

- Specific image processing to determine the linear dimensions with enough resolution. The specific applied processing consists of:

- Improvements of brightness and contrast by means of look up table (LUT) according to algorithms of linear, exponential or logarithmic transformations.

- Application of filters for convolution, smoothed and edge detection in the image (laplacian, differentiation, Sobel and Roberts) that allow extracting the specific dimension looked for. The specific routines used for this processing are:

- Clamp. It finds edges throughout a rectangular region of interest (ROI) that is located on the image to process and it measures the distance between the located edges.

- Circulate edge. Locates the intersection points between a set of lines with a circular area as an annular or the edge of a circular object, giving back the circle shape that better adapts to the found edges.

- Straight edges, locates points throughout an edge of an object and finds the line that better adjusts to the edge.
Figure 3 shows the process to extract the basic dimensions of the main volume of the coupler 3.a) and the narrow tubes 3.b).

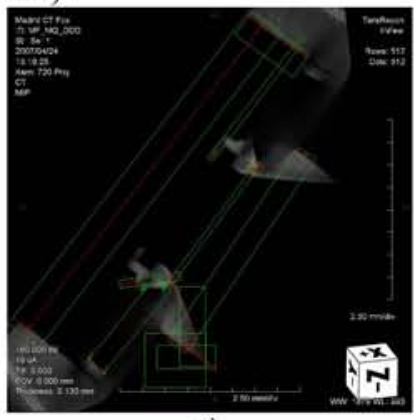

a)

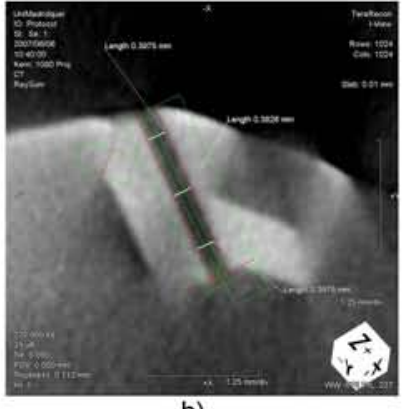

b)
Figure 3.- Non invasive X-Ray measurement system. Main cavities dimensions (a) and narrow tubes interconnection elements between volumes $V_{1}$ and $V_{3}(3 . b)$.

\section{B. Finite Element modelling}

The acoustic FEM/BEM modelling techniques require modelling the interior fluid of the coupler, i.e. the air contained in the inner cavities and interconnection elements (see figure 1). Figure 4 shows the geometry that has been simulated using LMS Virtual Lab Acoustic Version 7 (based in Sysnoise [9]) integrated in CATIA [10]. Mixed FEM/BEM modelling has been implemented. The volumetric cavities $V_{1}$, $\mathrm{V}_{2}, \mathrm{~V}_{3}$ have been modelled by means of FEM (fast and efficient in calculation), and interconnection elements, $L_{2} R_{2}$ and $L_{3} R_{3}$ by $B E M$ direct interior (it requires more time of calculation, but it allows to simulate thermal and viscosity losses, these effects are widely documented in the references [11], [12], [13], [14]).

\section{RESULTS}

The acoustic transference impedance has been measured using the reciprocity technique [15] in a laboratory with controlled environmental conditions. Later the internal and external dimensions from coupler prototype have been obtained by means of non-invasive techniques of measurement using X-ray inspection.

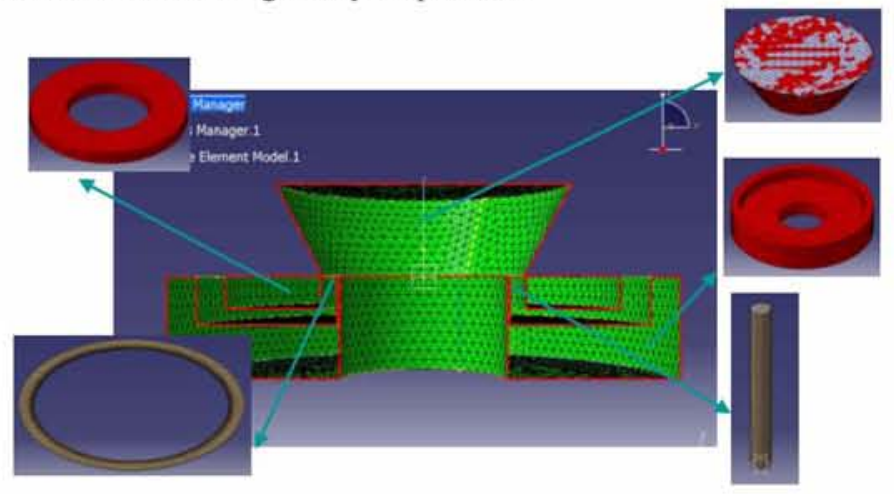

Figure 4.- Complete coupler FEM/BEM model in LMS virtual Lab. 
The dimensions obtained have been applied in the coupler's FEM/BEM finite element mixed model to obtain the acoustic impedance of the coupler.

Figure 5 shows the comparison among the data measured in the laboratory, the impedance values obtained in the simulation -these are in good agreement with the values defined in IEC60318-1 standard- and with the standard target values. There are differences in frequencies above $2 \mathrm{kHz}$ that reveal the limitations of the lumped param eter modelling. For these frequencies the FEM/BEM model results are more accurate and realistic than lumped parameter values provided in the standard. It is necessary to emphasize that threedimensional FEM/BEM model provides more detailed information about the physical behaviour in the interior of the acoustic coupler. So we can point out that:

- With this non-invasive inspection technique and FEM/BEM modelling it is possible to anticipate the behaviour of this type of couplers, in a repetitive and reliable way even before its manufacture and in very early stages of the design. It eliminates the necessity of expensive and complex processes to manufacture and characterize the device.

- The coupler key dimensions obtained with X-ray inspection make possible the obtaining of the acoustic impedance.

- Any complex geometry than cannot be analytically characterized, could be analyzed and used to improve this type of couplers, based on a FEM/BEM model.

- It would be possible to manufacture the coupler starting directly from FEM/BEM modelling. With this method the dimensions and geometry of the internal cavities of the coupler are perfectly determined. Traditional lumped modelling described in $\mathrm{ECC} 60318$ does not allow this.

In relation to the cost/complexity/performance analysis that can be summarize in table 1 , the technique using X-ray inspection plus Finite element modelling supposes "HighMedium" cost/complexity related to the current approach. However, this new method has some advantages respect to the drawbacks that the reciprocity technique present by itself.

For traditional approach, complex and expensive instrumentation equipment is need based on Bruel \&Kjaer PULSE measurement system for example, calibrated microphones and a climatic chamber is required. The jig for applying the stimulus to the coupler and extract the measurements have to be made without standarization by every laboratory itself.

Table 1.- Cost, complexity and performance comparison for traditional and proposed model.

\begin{tabular}{|c|c|c|c|}
\hline \hline Method used & Cost & Complexity & Perform ance \\
\hline $\begin{array}{c}\text { Reciprocity } \\
\text { Technique }\end{array}$ & Medium & High & Medium \\
\hline $\begin{array}{c}\text { X-ray+ FE } \\
\text { Model }\end{array}$ & High & Medium & High \\
\hline \hline
\end{tabular}

On the other hand, the proposed method can be made one time by any commercial X-ray inspection machine, with good resolution enough, for dimension extraction. Once mathematical finite element model is stated, any desired calculus may be done modifying fluid characteristics in the computer every time it is needed without compromise the cost and performance. The development was done one time and the results can be evaluated so much times you required depending on the study you want to focused on (without the needed of jig, microphones nor climatic chamber): mechanical tolerance dependence, temperature and presion variation, uncertainly estimation etc...The intercomparison problems between laboratories that arise with the traditional approach have no sense with the proposed methodology. It is stated that the presented method is a better and more flexible tool especially indicated for coupler design and allows acquiring deeper knowledge about the physical phenomena that take place in the fluid's coupler due to the post processing that can be done over the three dimensional map pressure response obtained.

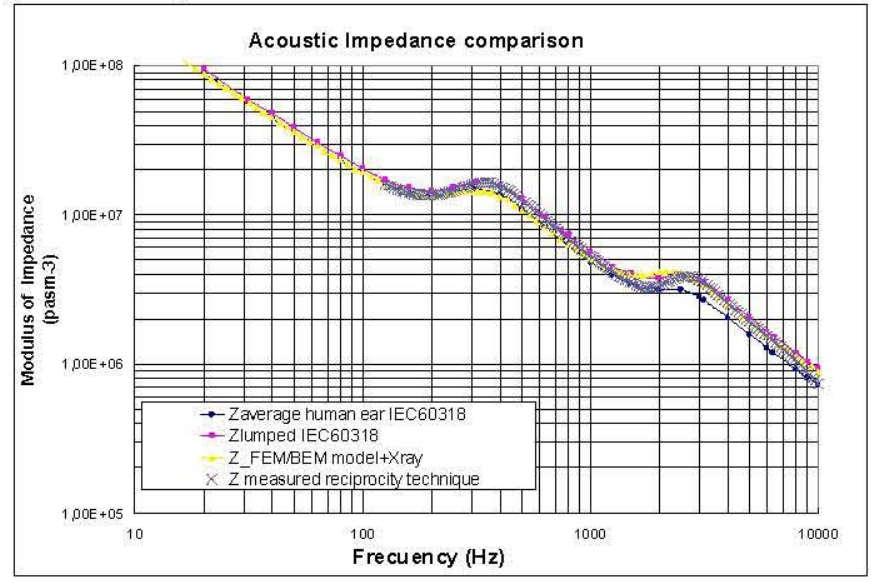

Figure 5.- Acoustic impedance comparison among IEC60318 data, traditional lumped model, average human ear, FEM/BEM model with $\mathrm{X}$-ray and reciprocity technique measurement.

\section{CONCLUSIONS}

The proposed approach supposes a reliable and trusty way of obtaining the acoustic transference impedance for acoustic couplers type I without the drawbacks that the reciprocity techniques has by itself and it supposes a good tool for the design of these kind of electroacoustic devices. More complex studies on these couplers could be carry out without the needed of the construction of a real device such as: mechanical tolerance influence, different environmental conditions dependencies, proposals of new geometries for the internal cavities and so on.

\section{ACKNOWLEDGMENT}

The author wishes to thank Comunidad de Madrid Government and Universidad Politécnica de Madrid for the grant CCG06-UPM/FI-312 received to develop the project. 


\section{REFERENCES}

[1] IEC 60318-1 Ed. 1.0 1998 Electroacoustics - Simulators of human head and ear- Part 1: Ear simulator for the calibration of supra-aural earphones.

[2] Sherwood. T.R. "Euromet Project 363 Harmonization of audiometry measurements within Europe. Final report". NPL Report CMAM 001. 1997.

[3] Pedroso M.A., Gerges S.N.Y., Gonçalves A.A. Jr.[UFSC/EMC Brazil]. Uncertainty in audiometer calibration. Metrologia, 2004, 41, $\mathrm{n}^{\circ} 1,1-7$

[4] M. Ruiz, B. Feuereisen, D. Machon and M. Recuero. Factors contributing to the uncertainty in circumaural earphone calibration for audiometric testing. Applied Acoustics, Volume 66, Issue 9, September 2005, Pages 1033-1048

[5] 29/633/CDV IEC/CD 60318-1 "Electroacoustics - Simulators of human head and ear - Part 1: Ear simulator for the calibration of supraaural and circumaural earphones". 2007

[6] Pierre G and Barham R. Finite Element Model of the IEC60318-1 artificial ear. National Physical Laboratory UK. Internoise 2001, The Hague. Holland.

[7] IEC 61094-2 Ed 2.0: 1996- Measurement Microphones. Part 2: Primary method for pressure calibration of laboratory standard microphone by the reciprocity technique.

[8] NI Vision for LabVIEW User Manual. 2007

[9] Virtual Lab REv6B and 7 A Manuals. Acoustic BEM/FEM Module de LMS

[10] CATIA V5 Manuals. 2005-2007. DASSAULT SYSTEMES.

[11] Ingard, U. "On the theory and design of acoustic Resonators", Acoustics Laboratory, Massachusetts Institute of Technology, Cambridge, Massachusetts (received June 19, 1953), The journal of The acoustic Society of America

[12] Ingard " Acoustic non linearity of an orifice", Acoustics Laboratory, Massachusetts Institute of Technology, Cambridge, Massachusetts (1967), The journal of The acoustic Society of America.

[13] Silvian, "Acoustic impedance of small orifice", Bell Telephone laboratories (1935). The journal of the acou stic Society of America.

[14] C.Zwicker and C.V. Kosten, "Sound absorbing material", Elsevier publishing (1949)

[15] Coupler calibration sheet for a commercial IEC60318 coupler, I2A2 Research Group- Madrid SPAIN 